\title{
,ane \\ CAN-Based Vibration Control for Networked Vehicle Active Suspension with Both Network-Induced Delays and Packet-Dropouts
}

\author{
Shi-Yuan Han *(D), Tong Liang, Jin Zhou and Yue-Hui Chen \\ Shandong Provincial Key Laboratory of Network Based Intelligent Computing, University of Jinan, \\ Jinan 250022, China; liangtong0606@163.com (T.L.); ise_zhouj@ujn.edu.cn (J.Z.); yhchen@ujn.edu.cn (Y.-H.C.) \\ * Correspondence: ise_hansy@ujn.edu.cn; Tel.: +86-531-8276-6503
}

Citation: Han, S.-Y.; Liang, T.; Zhou, J.; Chen, Y.-H. CAN-Based Vibration Control for Networked Vehicle Active Suspension with Both Network-Induced Delays and Packet-Dropouts. Electronics 2022, 11, 708. https://doi.org/10.3390/ electronics 11050708

Academic Editor: Maysam Abbod

Received: 20 January 2022

Accepted: 23 February 2022

Published: 25 February 2022

Publisher's Note: MDPI stays neutral with regard to jurisdictional claims in published maps and institutional affiliations.

Copyright: (C) 2022 by the authors. Licensee MDPI, Basel, Switzerland. This article is an open access article distributed under the terms and conditions of the Creative Commons Attribution (CC BY) license (https:// creativecommons.org/licenses/by/ $4.0 /)$.

\begin{abstract}
Ride comfort and driving safety are highly vulnerable to the undesirable excessive vibrations caused by road surface irregularities and the imperfect in-vehicle network (IVN). The main contribution of this paper consists of proposing a near-optimal vibration control approach for networked vehicle active suspension under irregular road excitations in a discrete-time domain, in which the uncertain time delay and packet dropout in CAN are taken into consideration. More specially, by virtue of two buffers of the sensor-to-controller network channel and the controller-to-actuator network channel in CAN, by introducing a designed state-transformation-based method, the original vibration control problem under the constraints of the irregular road excitations and imperfect CAN is transformed into a two-point boundary value (TPBV) problem without advanced and delayed items. After that, the near-optimal vibration control approach is presented to isolate the vehicle body from the road excitations and compensate the time delay and packet dropout from CAN synchronously. The stability condition of the networked vehicle active suspension under the proposed vibration controller is obtained based on the Lyapunov function. In numerous scenarios with different road roughnesses and network-induced time delays and packet dropouts, the simulation results illustrate the effectiveness and superiority of the proposed near-optimal vibration controller.
\end{abstract}

Keywords: networked vehicle active suspension; vibration control; time delay; packet dropout; irregular road excitation

\section{Introduction}

As a bridge between the automotive chassis and the irregular road surface, vehicle suspension is the core component in advanced driver assistance systems. Compared with passive and semi-active suspensions, vehicle active suspension with advanced vibration absorber technology can meet the increasing requirements for driving comfort and safety more effectively [1,2]. The embedded technology combined with a CAN-BUS in-vehicle network (IVN) system brings forward the concept of networked vehicle active suspension [3,4]. Many input and output signals should be transmitted via CAN-BUS IVN from the installed sensors to the control central then to the actuator for supervising and controlling the vehicle suspension system. The suspension maintenances with distributed computation benefit more effectively from the supplemented information. The modeling, analysis, and control of networked vehicle active suspension systems have received considerable attention in the last two decades [5-8].

The achievements of networked control systems (NCSs) put forward topics for further study of modeling and controller designs for networked vehicle active suspension. However, the network-induced time delay and packet dropout are still unavoidable issues for controller design, which means that the data of one or more of the data packets cannot reach the destination through the network. On the one hand, many studies focus on the networkinduced time delay. For example, by introducing a Markov jump-system framework for 
compensating the packet dropouts and time delays, Ref. [9] proposed a robust output feedback $H_{\infty}$ controller for a class of networked control systems with uncertain parameters and external disturbance; discussing time delays in two channels of a communication network, [10] proposed an event-based networked predictive controller for NCSs based on the measured outputs and the state predictions; a dynamic event-triggered $H \infty$ controller was designed for a class of nonlinear systems with unreliable communication channels and limited bandwidth in [11], in which the try-once-discard and flexible round-robin scheduling protocols were designed for assigning the unreliable communication channel. On the other hand, focusing on the packet dropout, a repeated game model is proposed in [12] to enhance IoT security and DT against selective forwarding (SF) attacks based on clustered wireless sensor networks (WSNs) and to preserve network stability; Ref. [13] investigated the optimal packet length and inter-node distance to be utilized in a SG over six different environments aiming at maximising the network lifetime to save power consumption; Ref. [14] proposed an event-triggered controller for a class of T-S systems based on the predictive packet; based on a compensating scheme for the packet dropout in the sensor-to-controller channel, a sliding mode controller was designed based on output information and state observer in [15]. Learning from the above studies, while discussing the controller design for networked vehicle active suspension, time delay, and packet dropout in the sensor-to-controller network channel and controller-to-actuator network channel must be taken into consideration synchronously.

Except for the influence of vehicle intrinsic factors, irregular road disturbance is usually viewed as the main external source of body shock and performance degradation. In most cases, road surface change in the vertical direction is approximated as a dynamic procedure involving a ground displacement power spectral density [16,17]. Meanwhile, the irregular road surface directly affects the ride comfort and driving safety, which involves three performance indices, including the suspension deflection, the tire deflection, and the sprung mass acceleration. Meanwhile, the performance trade-off between the ride comfort and driving safety is the most fundamental requirement of the process of designing the reasonable vibration controller [18]. Recently, many advanced optimal control theories, combined with disturbance rejection, have been developed for vehicle active suspension, such as stochastic optimal control for delayed vehicle active suspension [19], approximation optimal vibration control for nonlinear vehicle active suspension [20], preview-based technique for vehicle active suspension control [21], linear-quadratic-regulator-based control for vehicle active suspension [22], and so on. In general, while discussing the optimal vibration control problem with the network-induced time delay and packet dropout in CAN, the two-point boundary value (TPBV) problem with time-delay and time-advance items will be encountered, which makes it difficult to obtain the optimal analytic solution.

Motivated by integrating the optimal vibration control theory with the CAN-BUS technology, this work proposes a near-optimal vibration controller for vehicle active suspension under an imperfect CAN, which is suitable for speed sensors and acceleration sensors and can compensate for the external road disturbance and the internal network-induced time delay and packet dropout effectively. The main contributions of this work are as follows:

(1) By discussing the operating mechanism of vehicle active suspension under two introduced buffers in the sensor-to-controller network channel and the controller-toactuator network channel in imperfect CAN, a networked model of vehicle active suspension is formulated, in which the irregular road disturbance is viewed as an output from a constructed exosystem.

(2) By reformulating the original vibration control problem, a near-optimal vibration control approach is derived from an equivalent TPBV problem without the time-delay and time-advance items, based on a state-transformation-based method; correspondingly, the bound stability condition of networked vehicle active suspension is provided based on the Lyapunov function.

This work is organized as follows: Section 2 presents the model of networked vehicle active suspension. The near-optimal vibration control approach and stability analysis are 
described in detail in Section 3. Section 4 shows the validity and performance analyses based on the simulation results. The conclusion and future work are presented in Section 5.

\section{Modeling of Networked Vehicle Active Suspension in an Imperfect Controller Area Network}

\subsection{Networked Vehicle Active Suspension}

A typical quarter-networked vehicle active suspension under imperfect CAN with time delay and packet dropout is displayed in Figure 1. The system parameters of vehicle active suspension are listed as: $m_{s}$ and $z_{s}$ refer to the mass and displacement of sprung components; $m_{u}$ and $z_{u}$ stand for the mass and displacement of unsprung components; $k_{t}$ and $c_{t}$ represent the compressibility and damping of the pneumatic tire; and $c_{S}$ and $k_{S}$ are the damping and stiffness of vehicle suspension, respectively.

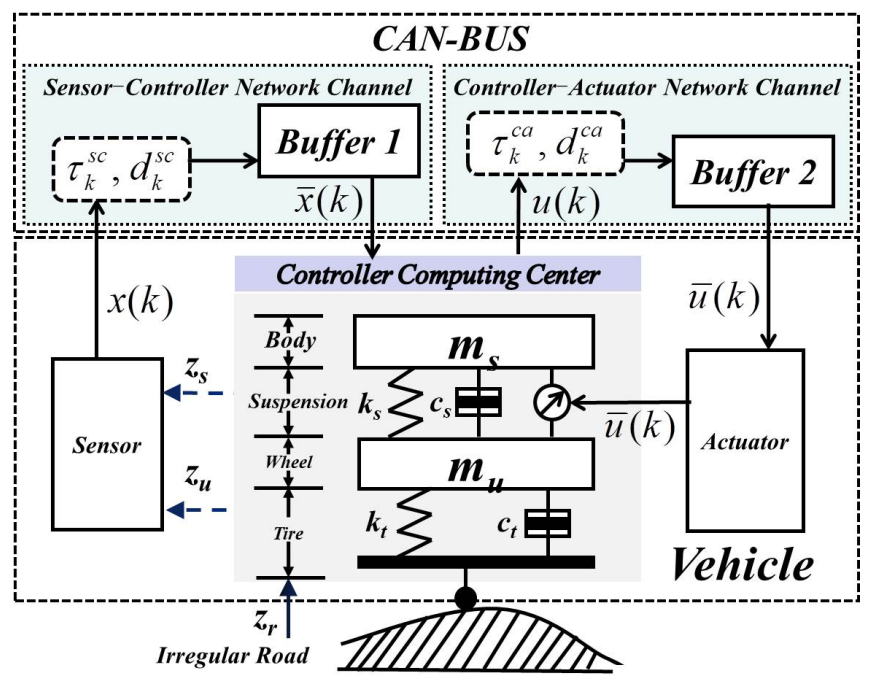

Figure 1. The quarter networked vehicle active suspension under imperfect CAN .

Based on the mechanical dynamic of the sprung and unsprung masses of vehicle active suspension in [1], the system state $x$ of vehicle suspension is defined as $x=\left[\begin{array}{llll}x_{1} & x_{2} & x_{3} & x_{4}\end{array}\right]^{T}$, which involves the suspension deflection $x_{1}=z_{s}-z_{u}$, the tire deflection $x_{2}=z_{u}-z_{r}$, the velocity $x_{3}=\dot{z}_{s}$ of sprung component, and the velocity $x_{4}=\dot{z}_{u}$ of unsprung component, respectively. By setting the sample period as $T$ in an ideal CAN without network-induced time delay and packet dropout, the normal form of networked vehicle active suspension in a discrete-time domain is described as in [7]:

$$
\begin{aligned}
& x(k+1)=A x(k)+B u(k)+D v(k), \\
& y_{c}(k)=C x(k)+E u(k), \\
& x(0)=x_{0},
\end{aligned}
$$

where $x_{0}$ is the initial system state; $v$ denotes the road disturbances related to the road irregularities, $Z_{r} ; u$ is the designed controller generated from advanced actuator, such as electric and hydraulic actuators; $y_{c}$ stands for the system output; and $A=e^{\bar{A} T}, B=$ $\int_{0}^{T} e^{\bar{A} t} \bar{B} d t$, and $D=\int_{0}^{T} e^{\bar{A} t} \bar{D} d t$, where 


$$
\begin{aligned}
& \bar{A}=\left[\begin{array}{cccc}
0 & 0 & 1 & -1 \\
0 & 0 & 0 & 1 \\
-\frac{k_{s}}{m_{s}} & 0 & -\frac{c_{s}}{m_{s}} & \frac{c_{s}}{m_{s}} \\
\frac{k_{s}}{m_{u}} & -\frac{k_{t}}{m_{u}} & \frac{c_{s}}{m_{u}} & -\frac{\left(c_{s}+c_{t}\right)}{m_{u}}
\end{array}\right], \bar{B}=\left[\begin{array}{c}
0 \\
0 \\
\frac{1}{m_{s}} \\
-\frac{1}{m_{u}}
\end{array}\right], \bar{D}=\left[\begin{array}{c}
0 \\
-1 \\
0 \\
\frac{c_{t}}{m_{u}}
\end{array}\right], \\
& E=\left[\begin{array}{c}
\frac{1}{m_{s}} \\
0 \\
0
\end{array}\right], C=\left[\begin{array}{cccc}
\frac{k_{s}}{m_{s}} & 0 & 0 & \frac{c_{s}}{m_{s}} \\
0 & 1 & 0 & 0
\end{array}\right] .
\end{aligned}
$$

Remark 1. The system output $y_{c}$ in (1) directly reflects the capabilities of improving the ride comfort and enhancing the driving safety, which involves the sprung mass acceleration, the suspension deflection, and tire deflection, and measures from the vertical velocity and acceleration sensors.

Considering an imperfect CAN, the network-induced time delay and packet dropout are taken into consideration in the two network channels, marked as $\tau^{S \mathcal{C}}(k)$ and $d^{S C}(k)$ in the sensor-to-controller network channel and $\tau^{c a}(k)$ and $d^{c a}(k)$ in the controller-to-actuator network channel, respectively, under the assumptions that the constraints of time delays are within $0 \leq \tau^{s c}(k)<m^{s c}$ and $0 \leq \tau^{c a}(k)<m^{c a}$, where $m^{s c}$ and $m^{c a}$ are the introduced upper bounds. Meanwhile, two buffers are employed to transfer the random time delays and packet dropouts into the constant quantities. More specially, for buffer 1 located between the sensor and the controller computing center, while the packet dropout $d^{s c}(k)=0$ at the current $k$ th sample period, the output of buffer 1 is set as $\bar{x}(k)=x\left(k-m^{s c}\right)$, otherwise, the packet $x\left(k-m^{s c}\right)$ is lost and $\bar{x}(k)=\bar{x}(k-1)$, while $d^{s c}(k)>0$. Similarly, for buffer 2 , located between the actuator and the controller computing center, while the packet dropout $d^{c a}(k)=0$ at the current $k$ th sample period, the output of buffer 2 is $\bar{u}(k)=u\left(k-m^{c a}\right)$, otherwise, the packet $u\left(k-m^{c a}\right)$ is lost and $\bar{u}(k)=\bar{u}(k-1)$, while $d^{c a}(k)>0$.

Based on the above mechanisms in CAN, the system model of networked vehicle active suspension is formulated as:

$$
\begin{aligned}
& x(k+1)=A x(k)+B \bar{u}(k)+D v(k), \\
& y_{c}(k)=C x(k)+E \bar{u}(k), \\
& \bar{u}(k)=\left\{\begin{array}{c}
u\left(k-m^{c a}\right), \quad d^{c a}(k)=0 \\
\bar{u}(k-1), \quad d^{c a}(k)>0
\end{array}\right. \\
& x(0)=x_{0}, \\
& \bar{u}(k)=0, \quad k=-m^{c a},-m^{c a}+1, \ldots, 0 .
\end{aligned}
$$

Assumption 1. The data packets transmitted in CAN are capsule time stamped with sample period T. While designing the two network channels, the allowed upper boundaries satisfy $m^{\text {sa }} \leq m^{c a}+T$.

\subsection{Irregular Road Disturbances}

For vehicle suspension, persistent irregular road disturbance is generally viewed as the primary origin of vibrations and specified as a random process involved with the following ground displacement PSD (power spectral density) under different road roughnesses [1], which is described as

$$
G_{d}(\Omega)= \begin{cases}G_{d}\left(\Omega_{0}\right)(2 \pi \Omega)^{-n_{1}}, & \Omega \leq \Omega_{0} \\ G_{d}\left(\Omega_{0}\right)(2 \pi \Omega)^{-n_{2}}, & \Omega>\Omega_{0}\end{cases}
$$

where $G_{d}\left(\Omega_{0}\right)$ is involved in the estimation of road roughness with constants $n_{1}$ and $n_{2}$; $\Omega$ represents the spatial frequency in the range of $\left[\beta_{1} \omega_{n} / v_{0}, \beta_{2} \omega_{n} / v_{0}\right]$ involved with the velocity $v_{0}$; and the vehicle natural frequency of $\omega_{n}=\sqrt{k_{s} / m_{s}}$, in which $0<\beta_{1}<1<\beta_{2}$. 
Considering the periodic characteristics of road irregularities, the road disturbance $v$ is formulated as

$$
v(t)=\dot{Z}_{r}(t)=0.2 v_{0} \sqrt{\frac{\pi G_{d}\left(\Omega_{0}\right)}{l}} \sum_{i=0}^{p-1} \cos \left[\left(\beta_{1} \omega_{n}+\frac{i 2 \pi v_{0}}{l}\right) t+\theta_{i}\right],
$$

where $\theta_{i} \in[0,2 \pi)$ is a random phase, and the frequency of irregular road disturbances is constrained by $p$ in the range of $\left[\left(\beta_{2}-\beta_{1}\right) \omega_{n} l / 2 \pi v_{0}+1,\left(\beta_{2}-\beta_{1}\right) \omega_{n} l / 2 \pi v_{0}+2\right)$. In the sample period $T$, the irregular road disturbance $v$ is generated from the output of the following exosystem in a discrete-time domain, which is described as

$$
\left\{\begin{array}{l}
w(k+1)=G w(k), \\
v(k)=F w(k)
\end{array}\right.
$$

where $w=\left[\begin{array}{llll}w_{1} & w_{2} & \cdots & x_{2 p}\end{array}\right]^{T}$ denotes the system state; $G=e^{\bar{G}_{v} T}$, where

$$
\begin{aligned}
& \tilde{G}=\operatorname{diag}\left\{-\left(\beta_{1} \omega_{n}\right)^{2}, \ldots,-\left(\beta_{1} \omega_{n}+(p-1) \frac{2 \pi v_{0}}{l}\right)^{2}\right\}, \\
& \bar{G}=\left[\begin{array}{ll}
0 & \mathrm{I} \\
\tilde{G} & 0
\end{array}\right], F=[\underbrace{0, \ldots, 0,}_{p} \underbrace{1, \cdots, 1}_{p}] .
\end{aligned}
$$

\subsection{The Network-Based Vibration Control Problem}

For improving the ride comfort and ensuring the road-holding ability, system output, $y_{c}$, must be restricted to small values with a light control force. However, the related three performance indicators conflict with each other. Meanwhile, due to the persistent road disturbance, the system state $x$, the system output $y_{c}$, and the control force $u(k)$ will not converge to zero synchronously. In order to describe the trade-off relationship among different performance requirements and control forces from an optimal control perspective, the following infinite-time average-performance index was selected, which is described as

$$
J=\lim _{N \rightarrow \infty} \frac{1}{N} \sum_{k=0}^{N}\left[y_{c}^{T}(k) Q y_{c}(k)+u^{T}(k) R u(k)\right] \text {, }
$$

where $Q=\operatorname{diag}\left(q_{1}, q_{2}, q_{3}\right)$, and $R$ is a positive definite matrix, in which $q_{i}(i=1,2,3)$ denote the trade-off weights for three performance indicators.

Thus, the objective of this paper is to design a near-optimal vibration controller $u^{*}(\cdot)$ for networked vehicle active suspension (3) under the constraint of irregular road disturbance (6) with respect to the performance index (8) that makes the performance index (8) obtain the minimum value, offsets the vibration caused by (6), and compensates the time delay and packet dropout in imperfect CAN.

\section{The Design of the Vibration Controller}

In this section, a state-transformation-based method is introduced, first to deal with the time delay in the actuator-to-controller network channel. After that, a near-optimal vibration controller is designed based on the transformed system model and the performance index to offset the road disturbance and compensate the imperfect CAN. Meanwhile, the bound stability condition of networked vehicle active suspension (3) is given under the proposed controller. 


\subsection{State-Transformation-Based Method}

While ignoring the packet dropout in imperfect CAN, the system model (3) of networked vehicle active suspension is rewritten as

$$
\begin{aligned}
& x(k+1)=A x(k)+B u\left(k-m^{c a}\right)+D v(k), \\
& y_{c}(k)=C x(k)+E u\left(k-m^{c a}\right), \\
& x(0)=x_{0} .
\end{aligned}
$$

By introducing the following state transformation,

$$
z(k)=x(k)+\sum_{i=k-m^{c a}}^{k-1} A^{k-m_{k}^{c a}-i-1} B u(i),
$$

Equation (9) is reformulated as the following, which is described as

$$
\begin{aligned}
& z(k+1)=A z(k)+\bar{B} u(k)+D v(k), \\
& y_{c}(k)=C z(k)+E u\left(k-m^{c a}\right)-\sum_{i=k-m^{c a}}^{k-1} A^{k-i-1} \bar{B} u(i), \\
& z(0)=x(0),
\end{aligned}
$$

where $\bar{B}=A^{-m^{c a}}$. Substituting the second formula of (11) into (8), (8) is reformulated as

$$
J=\lim _{N \rightarrow \infty} \frac{1}{N}\left\{2 z^{T}(k) C^{T} Q f(k, u)+f^{T}(k, u) Q f(k, u)+z^{T}(k) C^{T} Q C z(k)+u^{T}(k) R u(k)\right\},
$$

where $f(k, u)=E u\left(k-m^{c a}\right)-C \sum_{i=k-m^{c a}}^{k-1} A^{k-i-1} \bar{B} u(i)$.

Noticing that

$$
\begin{aligned}
& \lim _{N \rightarrow \infty} \frac{1}{N} \sum_{k=0}^{N} z^{T}(k) C^{T} Q f(k, u)=\lim _{N \rightarrow \infty} \frac{1}{N} \sum_{k=0}^{N}\left\{z^{T}(k) Q_{z u} u(k)+w^{T}(k) Q_{w u} u(k)+f_{z f}(k, u)\right\}, \\
& \lim _{N \rightarrow \infty} \frac{1}{N} \sum_{k=0}^{N} f^{T}(k, u) Q f(k, u)=\lim _{N \rightarrow \infty} \frac{1}{N} \sum_{k=0}^{N}\left\{u^{T}(k) \tilde{R}_{1} u(k)-2 f_{z f}(k, u)\right\},
\end{aligned}
$$

where

$$
\begin{aligned}
& Q_{z u}=\left(C A^{m^{c a}}\right)^{T} Q E-\sum_{i=1}^{m^{c a}}\left(\left(C A^{i}\right)^{T} Q C A^{i-1} \bar{B}\right), \\
& Q_{w u}=\left(\sum_{i=1}^{m^{c a}} C A^{m^{c a}-i} D F G^{i-1}\right)^{T} Q E-\sum_{i=1}^{m^{c a}} \sum_{j=0}^{m^{c a}-i}\left(\left(C A^{j} D F G^{i-1}\right)^{T} Q C A^{j+i-1} \bar{B}\right), \\
& \tilde{R}_{1}=E^{T} Q E-\sum_{i=1}^{m^{c a}}\left(\left(C A^{-i} B\right)^{T} Q C A^{-i} B\right), \\
& f_{z f}(k, u)=\left(\sum_{i=1}^{m^{c a}} C A^{-i} B u(k+i-1)\right)^{T} Q E u(k)+\sum_{i=1}^{m^{c a}} \sum_{j=i}^{m^{c a}}\left[\left(C A^{-j} B u(k+i-1)\right)^{T} \times Q C A^{i-j-1} B\right] u(k),
\end{aligned}
$$

substituting (13) into (12), the performance index (12) can be rewritten as

$$
J=\lim _{N \rightarrow \infty} \frac{1}{N} \sum_{k=0}^{N}\left\{z^{T}(k) C^{T} Q C z(k)+2 z^{T}(k) Q_{z u} u(k)+2 w^{T}(k) Q_{w u} u(k)+u^{T}(k) \tilde{R} u(k)\right\},
$$

where $\tilde{R}=R+\tilde{R}_{1}$.

Based on the above state-transformation-based method with the upper boundary $m^{c a}$ of the time delay, the original forms of time-delay system (9) and performance index (5) are reformed as the delay-free-equivalent ones. Thus, the random time delay $\tau^{c a}(k)$ in the controller-to-actuator network channel is easy to use to compensate for the pointto-point networked vehicle active suspension system. However, the network-induced packet dropouts are not discussed. Thus, the original vibration control problem could be reformulated to design a near-optimal vibration controller $u^{*}(\cdot)$ for transformed system (11) under the constraint of irregular road disturbance (6) with respect to the performance 
index (8) that makes the equivalent performance index (15) obtain the minimum value, offsets the vibration caused by (6), and compensates the packet dropout in imperfect CAN.

\subsection{The Design of the Near-Optimal Vibration Controller}

In this section, a near-optimal vibration controller is presented to eliminate the negative effects from the irregular road disturbance and the imperfect CAN.

Theorem 1. Considering the vibration control problem for networked vehicle active suspension Equation (3) under the constraints of road disturbances (6), network-induced time delay, and packet dropout with respect to performance index (8), a near-optimal vibration controller is designed as

$$
\begin{aligned}
& u^{*}(k)=-\tilde{R}^{-1}\left\{\left[Q_{w u}^{T}+\bar{B}^{T}\left(A-\bar{B} \tilde{R}^{-1} Q_{z u}^{T}\right)^{-T}\left(P_{1}+Q_{z u} \tilde{R}^{-1} Q_{w u}^{T}\right)\right] w(k)+\right. \\
& \left.\left[Q_{z u}^{T}+\bar{B}^{T}\left(A-\bar{B} \tilde{R}^{-1} Q_{z u}^{T}\right)^{-T}\left(P-Q_{1}\right)\right]\left[\bar{x}(k)+f_{\bar{x} u 1}(u, k)+f_{\bar{x} u 2}(u, k)+f_{\bar{u} w}(u, w, k)\right]\right\},
\end{aligned}
$$

where $f_{\bar{x} u 1}(u, k)=\sum_{i=1}^{m^{s a}} A^{i-1} B u\left(k+m^{s a}-m^{c a}-i\right), f_{\bar{x} u 2}(u, k)=\sum_{i=k-m_{k}^{c a}}^{k-1} A^{k-m_{k}^{c a}-i-1} B u(i)$, and $f_{\bar{u} w}(u, w, k)=\sum_{i=1}^{m^{s a}} A^{i-1} D G^{m^{s a}-i} F w(k) ; \bar{x}(k)$ denotes the output of buffer 1 with upper time delay $m^{s c}$ and packet dropout $d_{k}^{s c}$ in sensor-to-controller network channel, which is given by

$$
\bar{x}(k)=\left\{\begin{array}{l}
x\left(k-m^{s c}\right), \quad d^{s c}(k)=0 \\
\bar{x}(k-1), \quad d^{s c}(k)>0
\end{array}\right.
$$

$P$ is the unique positive definite solution of the Riccati matrix equation

$$
P-C^{T} Q C+Q_{z u} \tilde{R}^{-1} Q_{z u}^{T}=\left(A-\bar{B} \tilde{R}^{-1} Q_{z u}^{T}\right)^{T} P\left[I+\bar{B} \tilde{R}^{-1} \bar{B}^{T} P\right]^{-1}\left(A-\bar{B} \tilde{R}^{-1} Q_{z u}^{T}\right),
$$

and $P_{1}$ is the unique solution of the Stein matrix equation [23]

$$
\begin{aligned}
P_{1}+Q_{z u} \tilde{R}^{-1} Q_{w u}^{T}=(A- & \left.\bar{B} \tilde{R}^{-1} Q_{z u}^{T}\right)^{T} P\left[I+\bar{B} \tilde{R}^{-1} \bar{B}^{T} P\right]^{-1}\left(D F-\bar{B} \tilde{R}^{-1} Q_{w u}^{T}\right) \\
& +\left(A-\bar{B} \tilde{R}^{-1} Q_{z u}^{T}\right)^{T}\left[I-P\left[I+\bar{B} \tilde{R}^{-1} \bar{B}^{T} P\right]^{-1} \bar{B} \tilde{R}^{-1} \bar{B}^{T}\right] P_{1} G .
\end{aligned}
$$

Under the proposed near-optimal vibration controller (16), the actualized control force $\bar{u}(k)$ relates to the output of buffer 2 with packet dropout $d^{c a}(k)$ and upper time delay $m^{c a}$ in the controller-to-actuator network channel, which is described as

$$
\bar{u}(k)=\left\{\begin{array}{l}
u^{*}\left(k-m^{s c}\right), \quad d^{c a}(k)=0 \\
\bar{u}(k-1), \quad d^{c a}(k)>0
\end{array}\right.
$$

The proof is provided in Appendix A.

\subsection{Stability Analysis}

In this subsection, the bound stability condition of networked vehicle active suspension Equation (3) under the proposed near-optimal vibration controller (16) is analyzed.

Theorem 2 ([24]). The networked vehicle active suspension Equation (3) is asymptotically stable if there exist matrices $\bar{P} \in R^{4 \times 4}$ and $\bar{Q} \in R^{4 \times 4}$ satisfying $\left[\begin{array}{cc}\bar{Q}-\bar{P} & 0 \\ 0 & \bar{P}\end{array}\right]<0$.

From Theorem 2, it is proved that the stability of the original networked vehicle active system (3) can be guaranteed by the stability of the reformulated system (11). The proof is provided in Appendix A.

\section{Simulation Results and Discussion}

In this section, by employing a simple quarter-networked vehicle active suspension based on MATLAB/Simulink, the effectiveness of the proposed near-optimal vibration 
control approach based on state-transformation-based method will be verified based on simulation results.

The parameters of vehicle active suspension in Equation (1) and irregular road disturbance in Equation (5) are listed in Table 1. In the sample period $T=0.1 \mathrm{~s}$, the curve of the irregular road disturbance is displayed in Figure 2. Meanwhile, in order to discuss the performance in an imperfect in-vehicle network, the packet dropouts at the two network channels are simulated as two independent Bernoulli processes with probability distribution

$P\left\{d^{c a}(k)=1\right\}=\delta_{c a}, \quad P\left\{d^{c a}(k)=0\right\}=1-\delta_{c a}, \quad P\left\{d^{s a}(k)=1\right\}=\delta_{s a}, \quad P\left\{d^{s a}(k)=0\right\}=1-\delta_{s a}$,

where $\delta_{s c}$ and $\delta_{c a}$ denote the probability of the packet dropout in the sensor-to-controller channel and the controller-to-actuator channel, respectively. The trade-off weights in the quadratic performance index in Equation (8) are chosen as $q_{1}=8 \times 10^{7}, q_{2}=1.5 \times 10^{7}$, $q_{2}=2 \times 10^{7}$, and $R=212.05$.

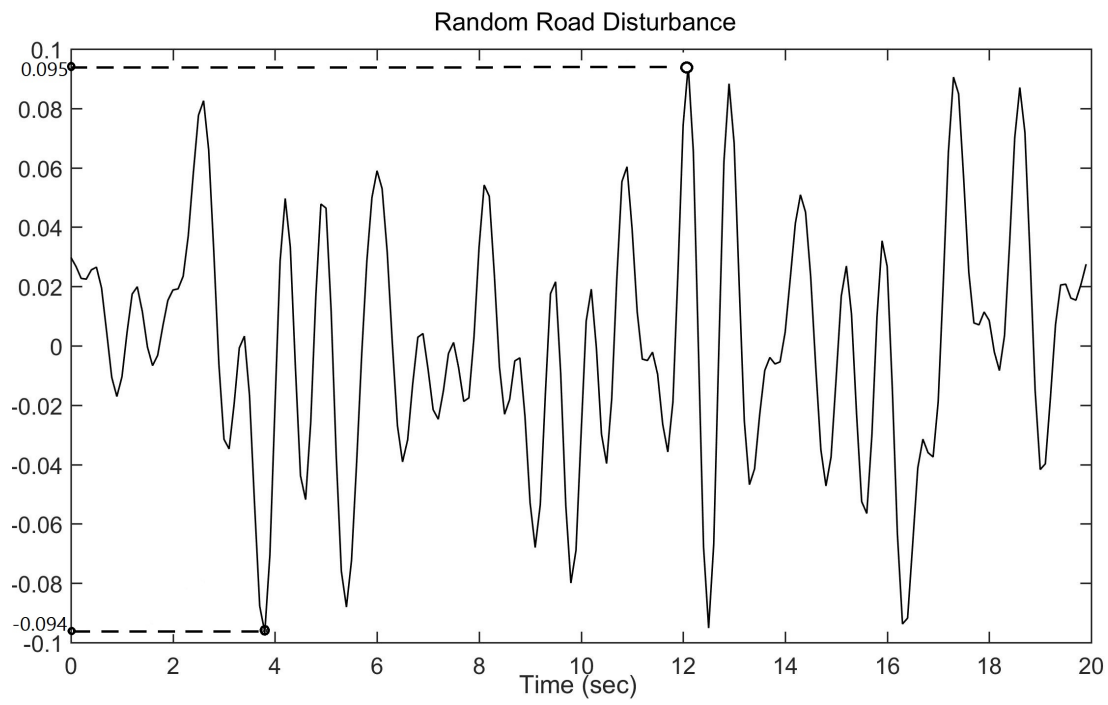

Figure 2. The curve of irregular road disturbances.

Table 1. Parameters of vehicle active suspension and road disturbance.

\begin{tabular}{lll}
\hline Parameters & Symbols & Values \\
\hline Sprung Mass of Vehicle Body & $m_{s}$ & $9527.6 \mathrm{~N}$ \\
Unsprung Mass of Vehicle Body & $m_{u}$ & $1113.3 \mathrm{~N}$ \\
Damping of Passive Suspension System & $c_{s}$ & $1095 \mathrm{Ns} / \mathrm{m}$ \\
Stiffness of Passive Suspension System & $k_{s}$ & $42,719.6 \mathrm{~N} / \mathrm{m}$ \\
Compressibility of Pneumatic Tyre & $k_{t}$ & $101,115 \mathrm{~N} / \mathrm{m}$ \\
Damping of Pneumatic Tyre & $c_{t}$ & $14.6 \mathrm{Ns} / \mathrm{m}$ \\
Vehicle Velocity & $v_{0}$ & $20 \mathrm{~m} / \mathrm{s}$ \\
Road Length & $l$ & $400 \mathrm{~m}$ \\
Constants of Road Roughness & $n_{1}, n_{2}$ & $2,1.5$ \\
Constants of Road Disturbance Frequency & $\beta_{1}, \beta_{2}$ & $0.5,5$ \\
\hline
\end{tabular}

In order to illustrate the effectiveness of compensating for the road disturbance and imperfect CAN, the control performance will be discussed under different time-delay bounds and packet dropout rates. The quadruple form $\left(m^{s c}, m^{c a}, \delta_{s c}, \delta_{c a}\right)$ is introduced to show the time-delay bounds and packet dropouts in two network channels. In order to illustrate the abilities of the proposed controller for compensating for the road disturbance and imperfect CAN, the RMS (root mean square) values are shown in Tables 2-4 under different road roughnesses, including PDS (packet drop statistics), SMA (sprung mass 
acceleration), SD (suspension deflection), TD (tyre deflection), proposed near-optimal vibration controller in Equation (16), and the value of performance index from Equation (8).

Table 2. RMS values of performance indicators under road roughness with $G_{d}\left(\Omega_{0}\right)=64 \times 10^{-6} \mathrm{~m}^{3}$.

\begin{tabular}{lllllll}
\hline$\left(\boldsymbol{m}^{s c}, \boldsymbol{m}^{c \boldsymbol{a}}, \boldsymbol{\delta}_{\boldsymbol{s c}}, \boldsymbol{\delta}_{\boldsymbol{c a}}\right)$ & PDS & SMA & SD & TD & Proposed Controller & $\boldsymbol{J}^{*}\left(\mathbf{1 0}^{\mathbf{7}}\right)$ \\
\hline Open-Loop Suspension System & - & 0.195 & 0.044 & 0.021 & - & 6.569 \\
$(0.2 s, 0.4 s, 0.98,0.95)$ & 15 & 0.087 & 0.018 & 0.012 & 325.832 & 7.322 \\
$(0.2 s, 0.4 s, 0.6,0.95)$ & 72 & 0.092 & 0.021 & 0.015 & 380.058 & 7.993 \\
$(0.2 s, 0.4 s, 0.98,0.6)$ & 80 & 0.098 & 0.025 & 0.018 & 340.067 & 7.843 \\
$(0.2 s, 0.4 s, 0.4,0.6)$ & 105 & 0.116 & 0.031 & 0.018 & 358.067 & 7.733 \\
$(0.3 s, 0.8 s, 0.98,0.95)$ & 13 & 0.089 & 0.024 & 0.014 & 330.064 & 7.983 \\
$(0.3 s, 0.8 s, 0.6,0.95)$ & 68 & 0.089 & 0.021 & 0.015 & 400.057 & 7.934 \\
$(0.3 s, 0.8 s, 0.98,0.6)$ & 74 & 0.105 & 0.031 & 0.018 & 360.057 & 8.230 \\
$(0.3 s, 0.8 s, 0.4,0.4)$ & 110 & 0.126 & 0.033 & 0.019 & 380.057 & $+18.9 \%$ \\
APR & - & $-48.5 \%$ & $-42.1 \%$ & $-23.2 \%$ & - & \\
\hline
\end{tabular}

Table 3. RMS values of performance indicators under road roughness with $G_{d}\left(\Omega_{0}\right)=256 \times 10^{-6} \mathrm{~m}^{3}$.

\begin{tabular}{lllllll}
\hline$\left(\boldsymbol{m}^{s c}, \boldsymbol{m}^{c \boldsymbol{a}}, \boldsymbol{\delta}_{\boldsymbol{s c}}, \boldsymbol{\delta}_{\boldsymbol{c} \boldsymbol{a}}\right)$ & PDS & SMA & SD & TD & Proposed Controller & $\boldsymbol{J}^{*}\left(\mathbf{1 0}^{\mathbf{8}}\right)$ \\
\hline Open-Loop Suspension System & - & 0.240 & 0.058 & 0.045 & - & 0.801 \\
$(0.2 s, 0.4 s, 0.98,0.95)$ & 12 & 0.112 & 0.033 & 0.028 & 515.063 & 1.123 \\
$(0.2 s, 0.4 s, 0.6,0.95)$ & 75 & 0.119 & 0.034 & 0.028 & 524.068 & 1.154 \\
$(0.2 s, 0.4 s, 0.98,0.6)$ & 81 & 0.188 & 0.047 & 0.036 & 581.141 & 1.734 \\
$(0.2 s, 0.4 s, 0.4,0.6)$ & 110 & 0.227 & 0.053 & 0.039 & 615.244 & 1.914 \\
$(0.3 s, 0.8 s, 0.98,0.95)$ & 15 & 0.166 & 0.034 & 0.030 & 530.256 & 1.174 \\
$(0.3 s, 0.8 s, 0.6,0.95)$ & 67 & 0.129 & 0.037 & 0.032 & 535.556 & 1.201 \\
$(0.3 s, 0.8 s, 0.98,0.6)$ & 78 & 0.141 & 0.044 & 0.038 & 562.169 & 1.834 \\
$(0.3 s, 0.8 s, 0.4,0.6)$ & 105 & 0.151 & 0.047 & 0.039 & 582.267 & +859 \\
APR & - & $-42.2 \%$ & $-32.7 \%$ & $-27.2 \%$ & - & $+8 \%$ \\
\hline
\end{tabular}

Table 4. RMS values of performance indicators under road roughness with $G_{d}\left(\Omega_{0}\right)=1024 \times 10^{-6} \mathrm{~m}^{3}$.

\begin{tabular}{lllllll}
\hline$\left(\boldsymbol{m}^{s c}, \boldsymbol{m}^{c \boldsymbol{a}}, \boldsymbol{\delta}_{\boldsymbol{s c}}, \boldsymbol{\delta}_{\boldsymbol{c} \boldsymbol{a}}\right)$ & PDS & SMA & SD & TD & Proposed Controller & $\boldsymbol{J}^{*}\left(\mathbf{1 0}^{\mathbf{8}}\right)$ \\
\hline Open-Loop Suspension System & - & 0.374 & 0.075 & 0.070 & - & 1.117 \\
$(0.2 s, 0.4 s, 0.98,0.95)$ & 16 & 0.192 & 0.050 & 0.046 & 712.362 & 2.521 \\
$(0.2 s, 0.4 s, 0.6,0.95)$ & 61 & 0.199 & 0.053 & 0.048 & 724.068 & 2.556 \\
$(0.2 s, 0.4 s, 0.98,0.6)$ & 74 & 0.236 & 0.060 & 0.056 & 771.122 & 3.837 \\
$(0.2 s, 0.4 s, 0.4,0.6)$ & 101 & 0.243 & 0.064 & 0.060 & 895.244 & 7.085 \\
$(0.3 s, 0.8 s, 0.98,0.95)$ & 19 & 0.198 & 0.052 & 0.045 & 791.269 & 2.584 \\
$(0.3 s, 0.8 s, 0.6,0.95)$ & 70 & 0.201 & 0.055 & 0.046 & 795.623 & 3.603 \\
$(0.3 s, 0.8 s, 0.98,0.6)$ & 75 & 0.251 & 0.061 & 0.060 & 922.213 & 3.239 \\
$(0.3 s, 0.8 s, 0.4,0.6)$ & 112 & 0.271 & 0.064 & 0.063 & 942.465 & $+154.9 \%$ \\
APR & - & $-40.2 \%$ & $-23.5 \%$ & $-24.3 \%$ & - & \\
\hline
\end{tabular}

From Tables 2-4, it can be noticed that the RMS values of SMA, SD, and TD could be scheduled to smaller values than open-loop ones under different road roughnesses, time-delay bounds, and packet dropout rates. Meanwhile, with the increase of road roughness, packet dropout rates, or time-delay bounds, the RMS values of the performance indicators and the performance index in Equation (8) always become worse correspondingly. Fortunately, the values of performance index $J$ in Equation (8) under the proposed controller in Equation (16) are still smaller than those of open-loop systems. Under different road roughnesses, the values of APR (average percentage reduction) represent the reduced percentages of the average values relative to the open-loop ones in Tables $2-4$, for example, the APR value of SD with a road roughness of $G_{d}\left(\Omega_{0}\right)=256 \times 10^{-6} \mathrm{~m}^{3}$ is 0.041 under eight simulation scenarios. Compared with 0.058 in open-loop vehicle suspension, the APR value of $\mathrm{SD}$ is reduced by $32.7 \%$. Corresponding to this, the APR value of the performance index $J$ 
in Equation (8) is $1.524 \times 10^{8}$, which increased by $87.4 \%$ compared with $0.801 \times 10^{8}$ in openloop vehicle suspension. The increment rate is mainly caused by the additional control consumption for compensating the irregular road disturbance and the network-induced time delays and packet dropouts.

More intuitively, considering the quadruple form $\left(\mathrm{m}^{s c}, \mathrm{~m}^{c a}, \delta_{s c}, \delta_{c a}\right)$ with $(0.3 s, 0.6 s$, $0.87,0.90)$ under different road roughnesses with $G_{d}\left(\Omega_{0}\right)=64 \times 10^{-6} \mathrm{~m}^{3}$ (B Grade), $G_{d}\left(\Omega_{0}\right)=256 \times 10^{-6} \mathrm{~m}^{3}$ (C Grade), and $G_{d}\left(\Omega_{0}\right)=1524 \times 10^{-6} \mathrm{~m}^{3}$ (E Grade), the comparison curves of the three performance indicators are shown in Figures 3-5, including SMA, $\mathrm{SD}$, and TD. Meanwhile, the comparison curves of the proposed near-optimal vibration controller $u^{*}(k)$ in Equation (16) under different simulation scenarios are displayed in Figure 6. From Figures 3-6, it can be summarized that, regardless of the conditions of the irregular road disturbance and imperfect $C A N$, the performance indicators could be converged within the acceptable range. At the same time, there is no significant increase in control consumption.

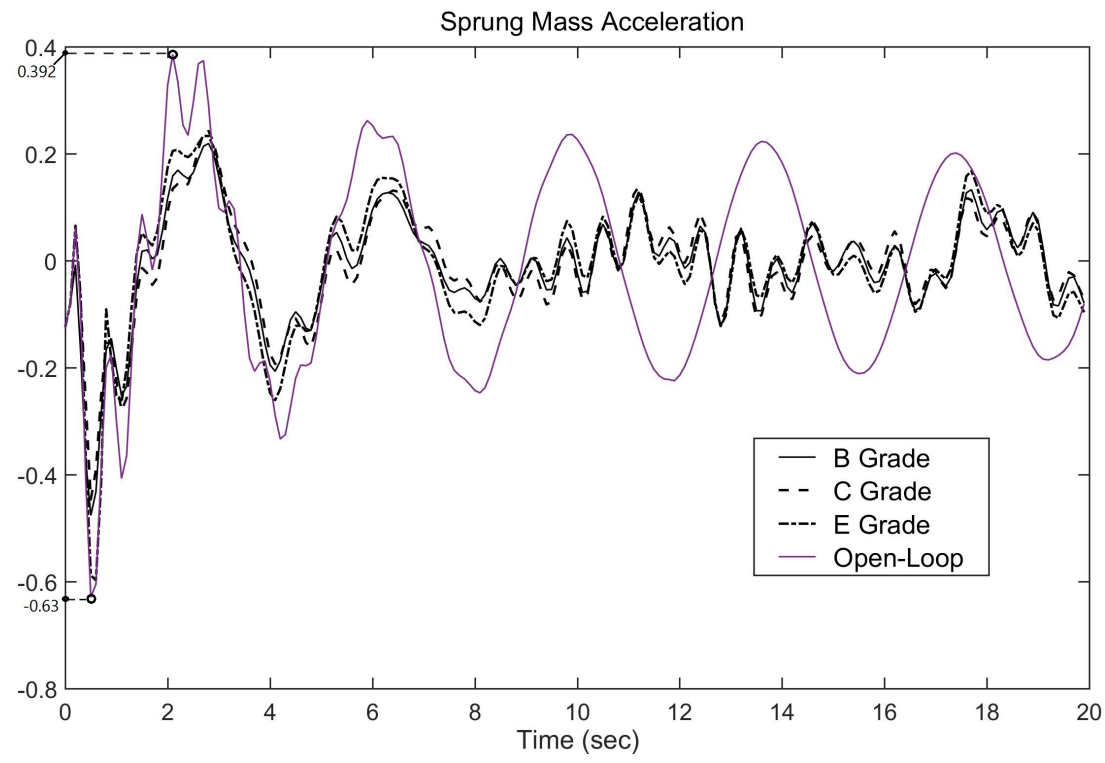

Figure 3. The comparison curves of SMA under different road roughnesses.

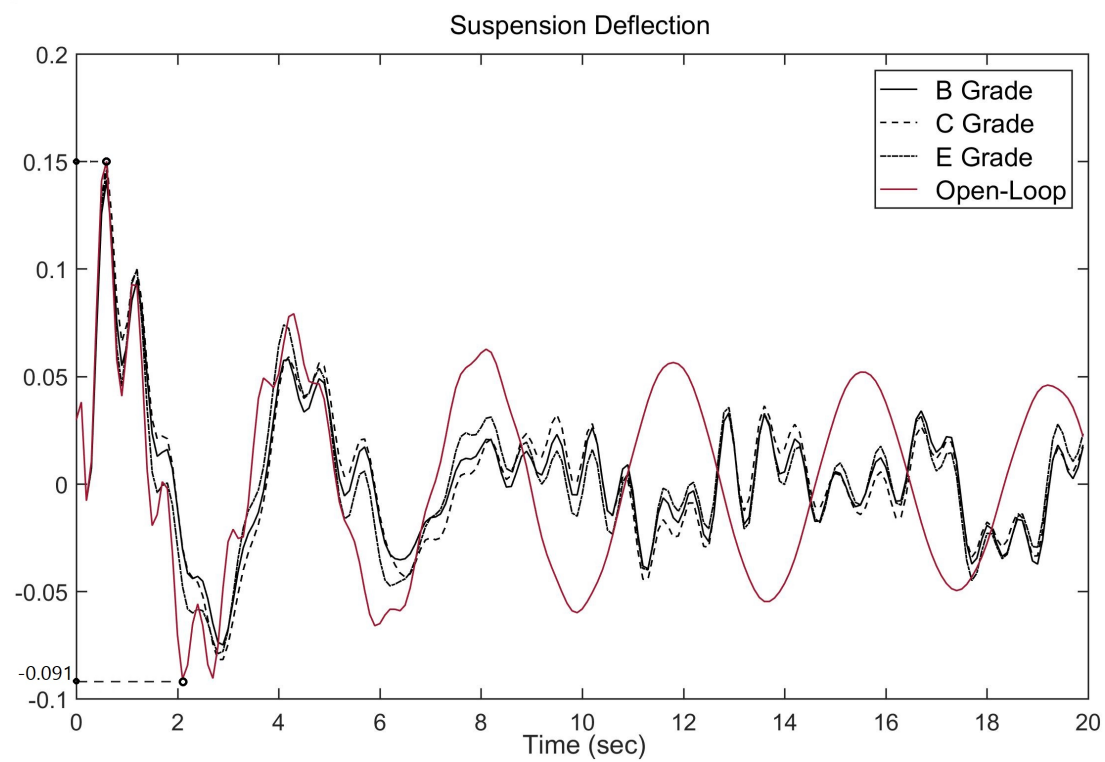

Figure 4. The comparison curves of SD under different road roughnesses. 


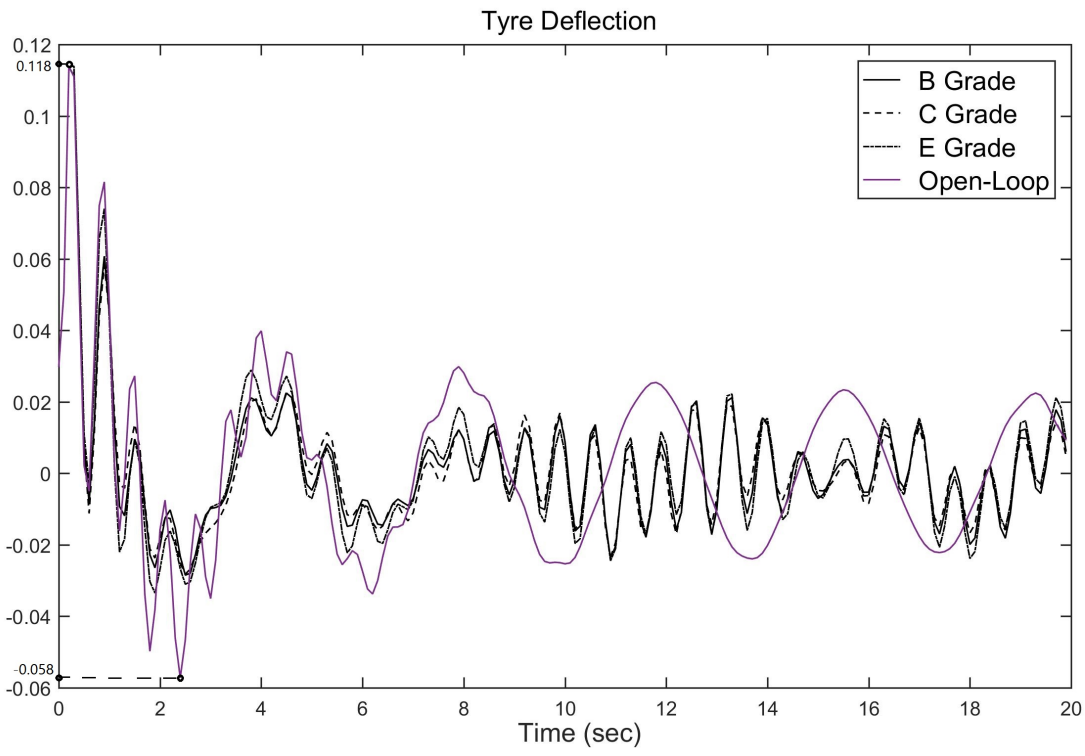

Figure 5. The comparison curves of TD under different road roughnesses.

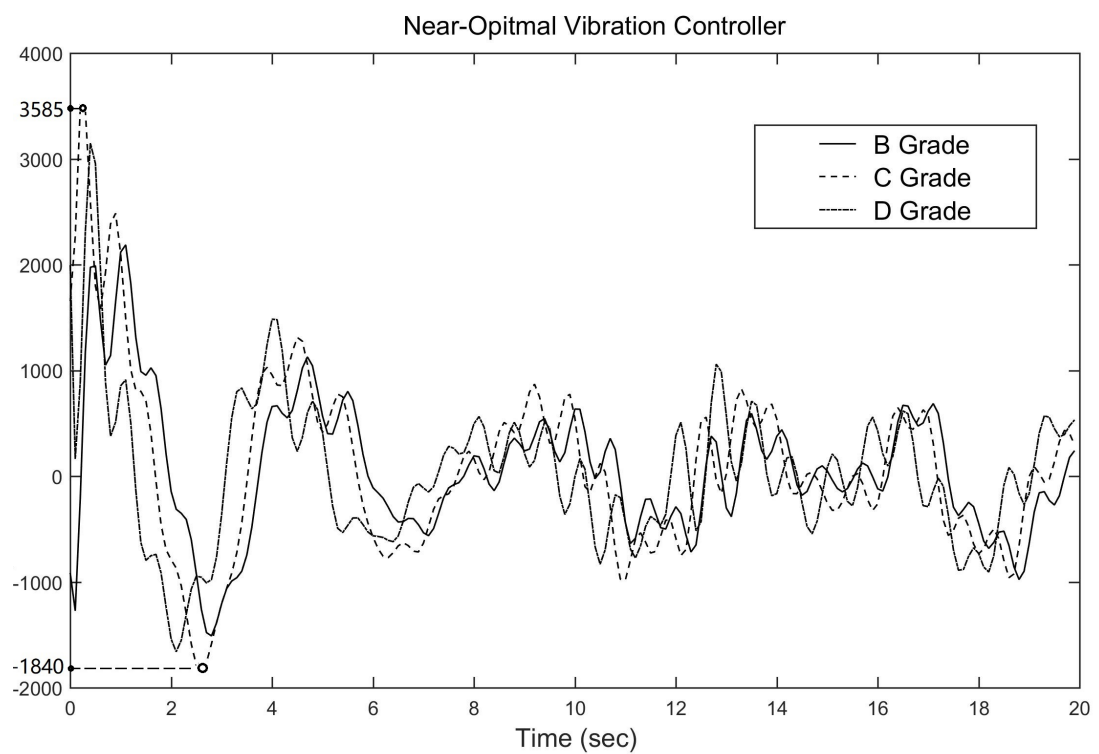

Figure 6. The comparison curves of the near-optimal vibration controller under different road roughnesses.

From Figures 3-6 and Tables 2-4, it can be observed that the proposed near-optimal vibration control approach can effectively compensate for the irregular road disturbance and imperfect CAN.

\section{Conclusions and Future Work}

The vibration control for a networked vehicle active suspension subject to persistent road disturbance and imperfect CAN has been investigated in this paper. By introducing two buffers in sensor-to-controller and controller-to-actuator network channels, the random network-induced time delay and packet dropout have become certainties. A near-optimal vibration controller has been obtained from a reformulated TPBV problem, which has been derived from the original vibration control problem by using a designed state-transformation-based method. From simulation results, the obtained vibration control scheme has shown the effectiveness of compensating the network-induced time delay and packet dropout, offsetting the vibration, and improving the control performance. 
In this paper, the model of vehicle active suspension is idealized as a linear system, where the dynamic properties of the active actuator are not considered. Moreover, the road disturbance is viewed as the output of the exosystem. It is interesting to investigate the vibration control issue of networked nonlinear vehicle active suspension with the estimation of road information and practical actuator constraints. Meanwhile, the sensors' power consumption is a very important metric in the process of suspension design. How to link the changes of suspension to adjusting the sampling period of sensors is an important issue related to saving the sensors' power consumption. The above discussions motivate our future work.

Author Contributions: Conceptualization, S.-Y.H. and Y.-H.C.; methodology, S.-Y.H. and T.L.; writing—original draft preparation, T.L.; writing—review and editing, S.-Y.H. and J.Z.; project administration, Y.-H.C. All authors have read and agreed to the published version of the manuscript.

Funding: This research was funded by the National Natural Science Foundation of China under Grants 61903156 and 61873324, the Natural Science Foundation of Shandong Province for Key Project under Grant ZR2020KF006, the Natural Science Foundation of Shandong Province under Grant ZR2019MF040, the University Innovation Team Project of Jinan under Grant 2019GXRC015, the Higher Educational Science and Technology Program of Jinan City under Grant 2020GXRC057, and the State Scholarship Fund of the China Scholarship Council.

Institutional Review Board Statement: Not applicable.

Informed Consent Statement: Not applicable.

Data Availability Statement: Not applicable.

Conflicts of Interest: The authors declare no conflict of interest.

\section{Abbreviations}

The abbreviations used in this paper:

CAN Controller Area Network

IVN In-Vehicle Network

TPBV Two-Point-Boundary-Value

NCSs Networked Control Systems

SF Selective Forwarding

WSNs Wireless Sensor Networks

PSD Power Spectral Density

RMS Root Mean Square

SMA Sprung Mass Acceleration

SD Suspension Deflection

TD Tyre Deflection

APR Average Percentage Reduction

\section{Appendix A}

The proofs of Theorems 1 and 2 are shown.

The Proof of Theorem 1. Applying the optimal control theory to the transformed system (11) with performance index (15), the optimal near-optimal vibration controller is designed as

$$
u^{*}(k)=-\tilde{R}^{-1}\left[Q_{z u}^{T} z(k)+Q_{w u}^{T} w(k)+\bar{B}^{T} \lambda(k+1)\right] .
$$

At the same time, a two-point boundary value (TPBV) problem with the solution $\lambda(k)$ is obtained as

$$
\left\{\begin{array}{l}
z(k+1)=\left(A-\bar{B} \tilde{R}^{-1} Q_{z u}^{T}\right) z(k)+\left(D F-\bar{B} \tilde{R}^{-1} Q_{w u}^{T}\right) w(k)-\bar{B} \tilde{R}^{-1} \bar{B}^{T} \lambda(k+1), \\
\left(C^{T} Q C-Q_{z u} \tilde{R}^{-1} Q_{z u}^{T}\right) z(k)+\left(A-\bar{B} \tilde{R}^{-1} Q_{z u}^{T}\right)^{T} \lambda(k+1)=\lambda(k)+Q_{z u} \tilde{R}^{-1} Q_{w u}^{T} w(k), \\
z(0)=x_{0}, \quad \lambda(\infty)=0 .
\end{array}\right.
$$


Introducing $\lambda(k)=P z(k)+P_{1} w(k)$, the TPBV problem in (22) is reformulated as

$$
\left\{z(k+1)=\left[I+\bar{B} \tilde{R}^{-1} \bar{B}^{T} P\right]^{-1}\left\{\left(A-\bar{B} \tilde{R}^{-1} Q_{z u}^{T}\right) z(k)+\left[D F-\bar{B} \tilde{R}^{-1} Q_{w u}^{T}-\bar{B} \tilde{R}^{-1} \bar{B}^{T} P_{1} G\right] w(k)\right\},\right.
$$

By substituting the second formula of (23) into (21), the optimal vibration control law $u^{*}(k)$ in $(21)$ can be rewritten as

$$
\begin{aligned}
u^{*}(k)=-\tilde{R}^{-1}\left\{\left[Q_{z u}^{T}+\bar{B}^{T}(A-\right.\right. & \left.\left.\bar{B} \tilde{R}^{-1} Q_{z u}^{T}\right)^{-T}\left(P-C^{T} Q C+Q_{z u} \tilde{R}^{-1} Q_{z u}^{T}\right)\right] z(k) \\
& \left.+\left[Q_{w u}^{T}+\bar{B}^{T}\left(A-\bar{B} \tilde{R}^{-1} Q_{z u}^{T}\right)^{-T}\left(P_{1}+Q_{z u} \tilde{R}^{-1} Q_{w u}^{T}\right)\right] w(k)\right\} .
\end{aligned}
$$

Meanwhile, based on the second formula of (23), the second formula of (22) can be reformulated as

$$
\begin{aligned}
\lambda(k)= & \left(C^{T} Q C-Q_{z u} \tilde{R}^{-1} Q_{z u}^{T}\right) z(k)+\left(A-\bar{B} \tilde{R}^{-1} Q_{z u}^{T}\right) \lambda(k+1)-Q_{z u} \tilde{R}^{-1} Q_{w u}^{T} w(k) \\
= & \left\{\left(A-\bar{B} \tilde{R}^{-1} Q_{z u}^{T}\right)^{T} P\left[I+\bar{B} \tilde{R}^{-1} \bar{B}^{T} P\right]^{-1}\left(A-\bar{B} \tilde{R}^{-1} Q_{z u}^{T}\right)+C^{T} Q C-Q_{z u} \tilde{R}^{-1} Q_{z u}^{T}\right\} z(k) \\
& +\left\{\left(A-\bar{B} \tilde{R}^{-1} Q_{z u}^{T}\right)^{T} P\left[I+\bar{B} \tilde{R}^{-1} \bar{B}^{T} P\right]^{-1}\left(D F-\bar{B} \tilde{R}^{-1} Q_{w u}^{T}\right)+\right. \\
& \left.\quad\left(A-\bar{B} \tilde{R}^{-1} Q_{z u}^{T}\right)^{T}\left[I-P\left[I+\bar{B} \tilde{R}^{-1} \bar{B}^{T} P\right]^{-1} \bar{B} \tilde{R}^{-1} \bar{B}^{T}\right] P_{1} G-Q_{z u} \tilde{R}^{-1} Q_{w u}^{T}\right\} w(k) .
\end{aligned}
$$

Notice that $\lambda(k)=P z(k)+P_{1} w(k)$, in comparison with the parameters of (25), the Riccati matrix Equation (18) and Stein Equation (19) are obtained. Thus the optimal vibration control law $u^{*}(k)$ in (24) is the computable formulation and is involved with the value of $z(k)$ in (9).

What follows, by using the outputs of Buffer 1 and Buffer 2, is that the networkinduced time delay with the upper boundary and packet dropout will be discussed. Based the first formula of (10), one obtains

$$
x\left(k+m^{s c}\right)=A^{m^{s a}} x(k)+\sum_{i=1}^{m^{s a}} A^{i-1} B u\left(k+m^{s a}-m^{c a}-i\right)+\sum_{i=1}^{m^{s a}} A^{i-1} D G^{m^{s a}}-i v(k) .
$$

While considering the network-induced packet dropout $d^{S C}(k)$ in the sensor-to-controller network channel, we obtain

$$
\bar{x}(k)=\left\{\begin{array}{l}
x\left(k-m^{s c}\right), \quad d_{k}^{s c}=0, \\
\bar{x}(k-1), \quad d_{k}^{s c}>0 .
\end{array}\right.
$$

Notice that $\bar{x}(k)=x\left(k-m^{s c}\right)$ and substituted (26) into $z(k),(10)$ can be rewritten as following equation

$$
\begin{aligned}
& z(k)=\bar{x}\left(k+m^{s c}\right)+\sum_{i=k-m_{k}^{c a}}^{k-1} A^{k-m_{k}^{c a}-i-1} B u(i)= \\
& A^{m^{s a}} \bar{x}(k)+f_{\bar{x} u 1}(u, k)+f_{\bar{x} u 2}(u, k)+f_{\bar{u} w}(u, w, k) .
\end{aligned}
$$

It should be pointed out that $z(k)$ in $(28)$ is computable at the $k$ th sampling period. By replacing $z(k)$ in (24) with (28), the proposed near-optimal vibration controller (16) is obtained. While considering the network-induced packet dropout $d^{c a}(k)$ in the controllerto-actuator network channel, one obtains

$$
\bar{u}(k)=\left\{\begin{array}{l}
u^{*}\left(k-m^{c a}\right), \quad d^{c a}(k)=0, \\
\bar{u}(k-1), \quad d^{c a}(k)>0
\end{array}\right.
$$

Thus, by integrating (27)-(29), the network-induced time delay and packet dropouts are compensated synchronously. Meanwhile, the designed near-optimal vibration controller (16) is physically realizable. This proof is completed. 
The Proof of Theorem 2. For the transformed system (11), a Lyapunov function is defined as

$$
V(z, k)=V_{1}(z, k)+V_{2}(z, k)=z^{T}(k) \bar{P} z(k)+\sum_{s=k-d^{c a}(k)}^{k-1} z^{T}(s) \bar{Q} z(s) .
$$

Defining the argument vector as $\bar{z}(k)=\left[z^{T}(k) w^{T}(k)\right]^{T}$, we obtain

$$
z(k) \triangleq\left[\begin{array}{ll}
I_{z} & 0
\end{array}\right] \bar{z}(k)=\Gamma_{1} \bar{z}(k) .
$$

By substituting (24) into the first formula of (11), one obtains

$$
z(k+1) \triangleq\left[\begin{array}{ll}
\Lambda_{1} & \Lambda_{2}
\end{array}\right] \bar{z}(k)=\Gamma_{2} \bar{z}(k) .
$$

where $\Lambda_{1}=A-\bar{B} \tilde{R}^{-1}\left[Q_{z u}^{T}+\bar{B}^{T}\left(A-\bar{B} \tilde{R}^{-1} Q_{z u}^{T}\right)^{-T}\left(P-Q_{1}\right)\right]$ and yields

$\Lambda_{2}=D F-\bar{B} \tilde{R}^{-1}\left[Q_{w u}^{T}+\bar{B}^{T}\left(A-\bar{B} \tilde{R}^{-1} Q_{z u}^{T}\right)^{-T}\left(P_{1}+Q_{z u} \tilde{R}^{-1} Q_{w u}^{T}\right)\right]$. From (32), one

$$
V_{1}(z, k+1)-V_{1}(z, k)=z^{T}(k+1) \bar{P} z(k+1)-z^{T}(k) \bar{P} z(k) \triangleq \bar{z}^{T}(k)\left(\Gamma_{2}^{T} \bar{P} \Gamma_{2}-\Gamma_{1}^{T} \bar{P} \Gamma_{1}\right) \bar{z}(k) .
$$

Considering that $d^{c a}(k+1)-d^{c a}(k) \leq 1$, we obtain

$$
\begin{aligned}
& V_{2}(z, k+1)-V_{2}(z, k)=\sum_{s=k+1-d^{c a}(k)}^{k} z^{T}(s) \bar{Q} z(s)-\sum_{s=k-d^{c a}(k)}^{k-1} z^{T}(s) \bar{Q} z(s) \\
& =z^{T}(k) \bar{Q} z(k)+\sum_{s=k+1-d^{c a}(k)}^{k-1} z^{T}(s) \bar{Q} z(s)-\sum_{s=k-d^{c a}(k)}^{k-1} z^{T}(s) \bar{Q} z(s) \leq z^{T}(k) \bar{Q} z(k) \\
& \triangleq \bar{z}^{T}(k) \Gamma_{1}^{T} \bar{Q} \Gamma_{1} \bar{z}(k) .
\end{aligned}
$$

Therefore, the transformed system model (11) is asymptotically stable under the proposed near-optimal vibration controller (16), if the following inequality holds

$$
\begin{aligned}
V(z, k+1)-V(z, k) & \leq \bar{z}^{T}(k)\left(\Gamma_{2}^{T} \bar{P} \Gamma_{2}-\Gamma_{1}^{T} \bar{P} \Gamma_{1}+\Gamma_{1}^{T} \bar{Q} \Gamma_{1}\right) \bar{z}(k) \\
& =\bar{z}^{T}(k)\left[\begin{array}{cc}
\Gamma_{1}^{T} & \Gamma_{2}^{T}
\end{array}\right]\left[\begin{array}{cc}
\bar{Q}-\bar{P} & 0 \\
0 & \bar{P}
\end{array}\right]\left[\begin{array}{c}
\Gamma_{1} \\
\Gamma_{2}
\end{array}\right] \bar{z}(k)<0 .
\end{aligned}
$$

It is obvious that (35) holds if there are positive definite matrices $\bar{P}$ and $\bar{Q}$ making $\left[\begin{array}{cc}\bar{Q}-\bar{P} & 0 \\ 0 & \bar{P}\end{array}\right]<0$, which means (11) is asymptotically stable.

We would like to mention that

$$
z(k)=x(k)+\sum_{i=k-m_{k}^{c a}}^{k-1} A^{k-m_{k}^{c a}-i-1} B u(i)=x(k)+\sum_{i=0}^{m^{c a}-1} A^{i-m^{c a}} B u(k-i-1) .
$$

Substituting (14) into (36), one obtains:

$$
\begin{aligned}
& \|x(k)\| \leq\|z(k)\| \\
& +m^{c a} \max _{0 \leq i \leq m^{c a}-1}\left\|A^{i-m^{c a}}\right\|\left\|B \tilde{R}^{-1}\left[Q_{z u}^{T}+\bar{B}^{T}\left(A-\bar{B} \tilde{R}^{-1} Q_{z u}^{T}\right)^{-T}\left(P-Q_{1}\right)\right]\right\|\|z(k-i-1)\| \\
& +m^{c a} \max _{0 \leq i \leq m^{c a}-1}\left\|A^{i-m^{c a}}\right\|\left\|B \tilde{R}^{-1}\left[Q_{w u}^{T}+\bar{B}^{T}\left(A-\bar{B} \tilde{R}^{-1} Q_{z u}^{T}\right)^{-T}\left(P_{1}+Q_{z u} \tilde{R}^{-1} Q_{w u}^{T}\right)\right]\right\|\|w(k-i-1)\| .
\end{aligned}
$$

Since $\|z(k)\|$ and $\|w(k)\|$ are bounded, $\|x(k)\|$ is bounded. Therefore, the networked vehicle active suspension (3) under the proposed near-optimal vibration controller (16) is asymptotically stable. This proof is completed. 


\section{References}

1. Ahmad, I.; Ge, X.; Han, Q.-L. Decentralized dynamic event-triggered communication and active suspension control of in-wheel motor driven electric vehicles with dynamic damping. IEEE/CAA J. Autom. Sin. 2021, 8, 971-986. [CrossRef]

2. Zheng, X.; Zhang, H.; Yan, H.; Yang, F.; Wang, Z.; Vlacic, L. Active full-vehicle suspension control via cloud-aided adaptive backstepping approach. IEEE Trans. Cybern. 2020, 50, 3113-3124. [CrossRef]

3. Fernando, M.-M.; Boada, B.L.; Boada, M.J.L.; Diaz, V. Ho dynamic output feedback control for a networked control active suspension system under actuator faults. Mech. Syst. Signal Process. 2022, 162, in press.

4. Zhong, X.-F.; Han, S.-Y.; Zhou, J.; Chen, Y.-H. Design of optimal disturbance attenuation controller for networked $T-S$ fuzzy vehicle active suspension with control delay. Int. J. Fuzzy Syst. 2019, 21, 676-684. [CrossRef]

5. Ge, X.; Ahmad, I.; Han, Q.-L.; Wang, J.; Zhang, X.-M. Dynamic event-triggered scheduling and control for vehicle active suspension over controller area network. Mech. Syst. Signal Process. 2021, 152, 10748. [CrossRef]

6. Wang, G.; Mohammed, C.; Chen, H.; Zhou, Z. Event-triggered control for active vehicle suspension systems with network-induced delays. J. Frankl. Inst. 2019, 365, 147-172. [CrossRef]

7. Han, S.-Y.; Zhou, J.; Chen, Y.-H.; Zhang, Y.-F.; Tang, G.-Y. Active fault-tolerant control for discrete vehicle active suspension via reduced-order observer. IEEE Trans. Syst. Man, Cybern. 2021, 51, 6701-6711. [CrossRef]

8. Shah, D.; Santos, M.M.D.; Chaoui, H.; Justo, J. Event-triggered non-switching networked sliding mode control for active suspension system with random actuation network delay. IEEE Trans. Intell. Transp. Syst. 2022, in press. [CrossRef]

9. Qiu, L.; Shi, Y.; Yao, F.; Xu, G.; Xu, B. Network-Based Robust $H_{2} / H_{\infty}$ control for linear systems with two-channel random packet dropouts and time delays. IEEE Trans. Cybern. 2015, 45, 1450-1462. [CrossRef]

10. Yang, R.; Yu, Y.; Sun, J.; Karimi, H.R. Event-based networked predictive control for networked control systems subject to two-channel delays. Inf. Sci. 2020, 524, 136-147. [CrossRef]

11. Zhao, G.; Hua, C.; Guan, X. Decentralized dynamic event-Triggered $H \infty$ control for nonlinear systems with unreliable communication channel and limited bandwidth. IEEE Trans. Fuzzy Syst. 2021, 29, 757-771. [CrossRef]

12. Abdalzaher, M.S.; Muta, O. A game-theoretic approach for enhancing security and data trustworthiness in IoT applications. IEEE Internet Things J. 2020, 7, 11250-11261. [CrossRef]

13. Elwekeil, M.; Abdalzaher, M.S.; Seddik, K. Prolonging smart grid network lifetime through optimising number of sensor nodes and packet length. Commun. IET 2019, 13, 2478-2484. [CrossRef]

14. Peng, C.; Wu, M.; Xie, X.P.; Wang, Y.L. Event-triggered predictive control for networked nonlinear systems with imperfect premise matching. IEEE Trans. Fuzzy Syst. 2018, 26, 2797-2806. [CrossRef]

15. Li, J.; Niu, Y. Output-feedback-based sliding mode control for networked control systems subject to packet loss and quantization Asian J. Control 2021, 23, 289-297. [CrossRef]

16. Hajidavalloo, M.R.; Li, Z.; Xia, X.; Zheng, M.; Zhuang, W. Cloud-assisted collaborative road information discovery with gaussian process: Application to road profile estimation. arXiv 2021, arXiv:2110.06977.

17. Xue, K.; Nagayama, T.; Zhao, B. Road profile estimation and half-car model identification through the automated processing of smartphone data. Mech. Syst. Signal Process. 2020, 142, 106722. [CrossRef]

18. Yatak, M.; Sahin, F. Ride Comfort-road holding trade-off improvement of full vehicle active suspension system by interval Type-2 fuzzy control. Eng. Sci. Technol. Int. J. 2021, 24, 259-270. [CrossRef]

19. Lei, J.; Jiang, Z. Active vibration control for nonlinear vehicle suspension with actuator delay via IO feedback linearization. Int. J. Control 2014, 87, 2081-2096.

20. Han, S.-Y.; Zhang, C.-H.; Tang, G.-Y. Approximation optimal vibration for networked nonlinear vehicle active suspension with actuator time delay. Asian J. Control 2017, 19, 983-995. [CrossRef]

21. Theunissen, J.; Tota, A.; Gruber, P.; Dhaens, M.; Sorniotti, A. Preview-based techniques for vehicle suspension control: A state-of-the-art review. Annu. Rev. Control 2021, 51, 206-235. [CrossRef]

22. Samsuria, E.; Sam, Y.M.; Ramli, L. Active suspension control by using linear quadratic regulator and sliding mode control techniques with optimisation. In Proceedings of the 2018 IEEE Conference on Systems, Process and Control (ICSPC), Melaka, Malaysia, 14-15 December 2018; pp. 135-140.

23. Stein, G.; Athans, M. The LQG/LTR procedure for multivariable feedback control design. IEEE Trans. Autom. Control 1987, 32, 105-114. [CrossRef]

24. Moore, J.; Sun, K. Lyapunov function construction for a class of discrete time-varying systems. IEEE Trans. Autom. Control 1969, 14, 595-595. [CrossRef] 